DRAFT VERSION JANUARY 14, 2019

Preprint typeset using $\mathrm{LAT}_{\mathrm{E}} \mathrm{X}$ style AASTeX6 v. 1.0

\title{
SYSTEMATIC PHYSICAL CHARACTERIZATION OF THE $\gamma$-RAY SPECTRA OF 2FHL BLAZARS
}

\author{
Jacobus P. VAn den Berg and Markus Böttcher \\ Centre for Space Research, North-West University, Potchefstroom, 2531, South Africa \\ Alberto Domínguez and Marcos LóPez-Moya \\ Grupo de Altas Energías and IPARCOS, Universidad Complutense de Madrid, E-28040 Madrid, Spain
}

\begin{abstract}
We test different physically motivated models for the spectral shape of the $\gamma$-ray emission in a sample of 128 blazars with known redshifts detected by the Fermi Large Area Telescope (LAT) at energies above $50 \mathrm{GeV}$. The first nine years of LAT data in the energy range from $300 \mathrm{MeV}$ to $2 \mathrm{TeV}$ are analyzed in order to extend the spectral energy coverage of the 2FHL blazars in our sample. We compare these spectral data to four leptonic models for the production of $\gamma$-rays through Compton scattering by a population of electrons with different spectral shapes. In the first three models we consider Compton scattering in the Thomson regime with different acceleration mechanisms for the electrons. In the fourth model we consider Compton scattering by a pure power law distribution of electrons with spectral curvature due to scattering in the Klein-Nishina regime. The majority of blazar $\gamma$-ray spectra are preferentially fit with either a power law with exponential cut-off in the Thomson regime or a power law electron distribution with Compton scattering in the Klein-Nishina regime, while a log-parabola with a low-energy power-law and broken power-law spectral shape in the Thomson regime appears systematically disfavoured, which is likely a consequence of the restriction to pure Thomson scattering which we imposed on those models. This finding may be an indication that the $\gamma$-ray emission from FSRQs in the 2FHL catalog is dominated by Compton scattering of radiation from the dusty torus, while in the case of BL Lac objects, it is dominated by synchrotron self-Compton radiation.
\end{abstract}

Keywords: galaxies: active - galaxies: jets — gamma-rays: galaxies — radiation mechanisms: nonthermal - relativistic processes

\section{INTRODUCTION}

Blazars are a subclass of active galactic nuclei (AGN) whose jets are oriented at a small angle with respect to an observer's line of sight. This geometry leads to relativistic aberration effects and Doppler boosting along the jet direction. Blazars are characterized by strong non-thermal emission across the electromagnetic spectrum, rapid variability, and high optical polarization. This is the brightest and most numerous source class in the persistent extragalactic $\gamma$-ray sky (Acero et al. 2015).

The spectral energy distributions (SEDs) of blazars are characterized by two broad, non-thermal components. It is widely accepted that the low-energy com-

24182869@nwu.ac.za, Markus.Bottcher@nwu.ac.za

alberto@gae.ucm.es, marcos@gae.ucm.es ponent is due to synchrotron radiation (SR) of relativistic electrons (and possibly positrons) accelerated in the blazar jet. For the high-energy component, both leptonic and hadronic origins are possible (e.g., Böttcher et al. 2013). In leptonic models, the X-ray and $\gamma$-ray emission is caused by inverse Compton (IC) scattering of low-energy photons by the same population of electrons which produced the SR. In this case, the shape of the $\gamma$-ray spectrum is directly related to the energy distribution of the accelerated electrons. This correlation is straightforward in the case of Compton scattering in the Thomson regime, but more complex in the KleinNishina regime (Böttcher et al. 2012; Dermer \& Menon 2012). Whether $\gamma$-ray production by Compton scattering proceeds in the Thomson or Klein-Nishina regime, depends critically on the characteristic target photon energy. If the target photons originate from the cospatially produced synchrotron emission (typically peak- 
ing in the infrared to optical regime in the co-moving frame, leading to synchrotron self-Compton, SSC, emission) or from a dusty torus around the central accretion flow (with target photons in the infrared, leading to external Compton on dust torus emission), then the Compton scattering to $\mathrm{GeV} \gamma$-ray energies typically occurs in the Thomson regime. In the case that the target photons originate externally from the Broad Line Region (dominated by optical to ultraviolet photons in the stationary frame of the AGN, leading to external Compton on BLR emission), then the Compton scattering to $\mathrm{GeV}$ energies typically occurs in the Klein-Nishina regime. A deviation of the $\gamma$-ray spectra of blazars from a pure power law may thus be caused either by an underlying electron population that deviates from a pure power law, and/or by the transition of the Compton scattering process from the Thomson to the Klein-Nishina regime towards higher $\gamma$-ray energies.

Evidence for non-power law electron distributions has been found in the synchrotron continuum spectra of blazars. Landau et al. (1986) showed that the lowenergy peak of fifteen (out of a sample of eighteen) blazars are well fitted by a log-parabolic form. These authors showed that an energy dependent probability of stochastic acceleration, specifically if the acceleration probability decreases with increasing energy, leads naturally to an electron distribution with a log-parabolic form. In this context, the curvature of the spectra is not simply due to energy losses but is rather a direct consequence of the acceleration mechanism. This result was verified for the case of Mrk 421 (Massaro et al. 2004; Tramacere et al. 2007) and for other BL Lac objects (Tramacere et al. 2011, and references therein). In particular, Massaro et al. (2004) also showed that a power law with exponential cut-off does not fit the synchrotron spectrum of Mrk 421 satisfactorily. This spectral shape might be expected if some limiting process is present in an acceleration mechanism such as diffusive shock acceleration (DSA; e.g., Kirk \& Heavens 1989; Ellison et al. 1990; Summerlin \& Baring 2012).

It has long been also recognized that the $\gamma$-ray spectra of blazars cannot be fitted by a simple power law (Abdo et al. 2010a). This is expected in the framework of leptonic models, where the same electron population produces both the synchrotron and $\gamma$-ray emission through Compton scattering (e.g., Böttcher 2007). Note that the shape of the underlying particle distribution will determine the shape of the Compton $\gamma$-ray spectrum (see Section 3).

Being able to characterise the high-energy spectra of a large sample of blazars may allow us to probe the underlying relativistic electron distribution and the characteristic energy of target photons for Compton scattering. Therefore, this methodology is a tool to diagnose the physical mechanisms of particle acceleration in the jets of blazars.

In this work, we compare the broad-band $\gamma$-ray spectra of 128 blazars selected from the Second Catalog of Hard Fermi-LAT Sources (the 2FHL catalog) with physically motivated models, over an energy range of almost four orders of magnitude, in an attempt to systematically characterize the spectral shape of the high-energy turnover. We stress that we do not aim to constrain physical parameters, but only investigate statistically the underlying physical processes.

The outline of this paper is as follows. Section 2 describes our blazar sample and data analysis. In Section 3 , the high-energy $\gamma$-ray spectra for the four theoretical models are derived. We describe our fitting methodology in Section 4 leading to the results presented in Section 5. Finally, Section 6 contains a summary and discussion of the results.

\section{SOURCE SAMPLE AND DATA ANALYSIS}

In this Section, we describe our blazar sample and data analysis. Figure 1 shows four examples of the spectral results from our analysis.

\subsection{Description of the Sample}

Our sample includes all the 128 blazars with known redshifts from the Fermi Large Area Telescope (LAT) 2FHL catalog (sources detected at energies larger than 50 GeV, Ackermann et al. 2016; Domínguez \& Ajello 2015). The redshifts range from $z=0.004283$ (M87) to $z=2.1$ (MG4 J00800+4712), with the median of the distribution at $z=0.215$.

Blazars tend to be divided in two main populations according to properties of their optical spectra. There are (almost) featureless objects known as BL Lac blazars, and flat-spectrum radio quasars (FSRQs), typically characterized by broad emission lines (Urry \& Padovani 1995). According to the blazar sequence, which is empirically derived, BL Lacs are characterized, on average, by harder $\gamma$-ray spectra and lower luminosity than FSRQs (Fossati et al. 1998; Ghisellini et al. 2017). Our sample contains 106 BL Lacs (with or without prominent galaxy emission), 10 FSRQs, 4 blazars of uncertain type (BCUs), and some radio galaxies and other types of AGN.

Another blazar classification methodology is motivated by the frequency at which their synchrotron peak is located. This characteristic frequency, which is provided in the $2 \mathrm{FHL}$, classifies these sources as lowsynchrotron peak (LSP), intermediate-synchrotron peak (ISP), and high-synchrotron peak (HSP) blazars with their synchrotron peak frequency at $\log _{10}\left(\nu_{\text {peak }}^{s}\right)<14$, $14<\log _{10}\left(\nu_{\text {peak }}^{s}\right)<15, \log _{10}\left(\nu_{\text {peak }}^{s}\right)>15$, respectively, with $\nu_{\text {peak }}^{s}$ given in units of $\mathrm{Hz}$. The 2FHL blazars 
are mostly catalogued as HSP BL Lacs (see Figure 8 in Ackermann et al. 2016). The exact numbers in our sample are 33 LSP, 12 ISP, and 82 HSP blazars (there is one source without clear classification due to a poorly sampled SED).

\subsection{Data Analysis}

The first nine years (450 weeks, from MJD 56048 to MJD 57772) of Fermi-LAT data were analyzed in the energy range from $300 \mathrm{MeV}$ to $2 \mathrm{TeV}$ in order to extend the energy spectral coverage of the $2 \mathrm{FHL}$ blazars in our sample. We analyzed this data set using the P8R2_SOURCE_V6 instrument response functions and the Fermi Science Tools version v10r0p5. Events were selected within a circular region of interest (ROI) of $15^{\circ}$ centred at the 2FHL source position. We selected "Source" class events (evclass $=128$ and evtype $=3$ ) that were recorded only when the telescope was in nominal science mode. To reject the background coming from the Earth's limb, we selected photons with a zenith angle $\leq 90^{\circ}$. For the spectral reconstruction, a binned likelihood analysis was performed making use of the pyLikelihood python module of the Fermi tools. We started by including all the sources from the Third Fermi Source Catalog (3FGL, Acero et al. 2015) in the spectral-spatial model. All the 3FGL sources were assumed to have spectral types as suggested in the catalog. The spectral parameters for sources with a significance larger than $5 \sigma$ and located less than $5^{\circ}$ away from the ROI centre were left free. We also let the normalization factor of the isotropic (iso_P8R2_SOURCE_V6_v06.txt) and Galactic (gll_iem_v06.fits) background models be free. For the rest of the sources all the parameters were left fixed to their catalog value. Finally, all sources with significance lower than $2 \sigma$ were removed from the model. For the calculation of the spectral points, we repeated the procedure in each energy bin using a power law with the normalization factor free and the spectral index fixed to 2 (where the spectral index $\Gamma$ is defined as $\propto E^{-\Gamma}$ ). Whenever the significance of the spectral point was less than $1.5 \sigma$, an upper limit was calculated instead.

\section{LEPTONIC MODELS OF $\gamma$-RAY EMISSION}

In this Section, we describe and derive our physically motivated models for the $\gamma$-ray emission in jets. In this study, we only consider leptonic emission processes, in which $\gamma$-rays are produced by Compton scattering off relativistic electrons. The recent possible association of the blazar TXS 0506+056 with the track-like EHE neutrino event IceCube-170922A (Aartsen et al. 2018a) as well as a possible neutrino flare in 2014 - 2015 from the same source (Aartsen et al. 2018b), suggest that at least in some blazars, hadronic emission processes play a role. These could lead to more complicated spectral fea- tures than considered here, due to the multi-component nature of the $\gamma$-ray emission (proton synchrotron + secondary-electron synchrotron from cascades + muon synchrotron + pion synchrotron), and their study is beyond the scope of this paper.

\subsection{Theoretical Background}

The first model is based on an electron distribution given by a power law with exponential cut-off, consistent with radiation-reaction-limited first-order Fermi acceleration (e.g., DSA). The second model is based on a log-parabolic electron distribution, which would be indicative of stochastic acceleration of electrons in the jet as suggested by Massaro et al. $(2004,2006)$ and Tramacere et al. (2007, 2011). The fourth model uses an electron distribution described by a broken power law, which could result from different acceleration / cooling mechanisms dominating in different energy ranges. The resulting Compton $\gamma$-ray spectra of these models are derived in the Thomson regime, so that the $\gamma$-ray spectrum directly reflects the underlying electron distribution. The last model assumes a simple power law electron distribution with the main spectral features caused by the decrease of the Compton cross section in the highenergy Klein-Nishina regime. It is well established that Compton-scattering scenarios are generally well suited to reproduce the $\gamma$-ray spectra of blazars with reasonable physical parameters. We therefore do not evaluate the normalization of the resulting Compton spectra in detail, as we do not attempt to constrain specific parameters of the physical setup with our fits, but merely investigate the spectral shape.

For given electron and synchrotron photon distributions, $n_{e}\left(\gamma_{e} ; \Omega_{e}\right)$ and $n_{\mathrm{ph}}\left(\epsilon_{\mathrm{ph}} ; \Omega_{\mathrm{ph}}\right)$ respectively, the observed Compton flux $\nu F(\nu)=\epsilon F_{\epsilon}$ as a function of the up-scattered photons' dimensionless photon energy $\epsilon=h \nu /\left(m_{e} c^{2}\right)$, is given in terms of the Compton cross section. In the Thomson regime, the differential Compton cross section can be approximated by a delta function (see Böttcher et al. 2012; Dermer \& Menon 2012), where a target photon of dimensionless energy $\epsilon_{\mathrm{ph}}$ is up-scattered by an electron with Lorentz factor $\gamma_{e}=$ $\left(1-\beta_{e}^{2}\right)^{-1 / 2}$, interacting at an angle $\mu=\cos \theta$, to a scattered photon energy of $\epsilon_{\mathrm{sc}}=\gamma^{2}\left(1-\beta_{e} \mu\right) \epsilon_{\mathrm{ph}}$. It is assumed for simplicity that the electron and target photon distributions are isotropic. As the shape of the scattered photon spectrum is dominated by the shape of the electron spectrum, one can approximate any narrow target photon distribution (such as, e.g., the BLR or dust-torus infrared radiation) as mono-energetic, so that $n_{\mathrm{ph}}\left(\epsilon_{\mathrm{ph}}\right) \approx n_{\mathrm{ph} ; 0} \delta\left(\epsilon_{\mathrm{ph}}-\epsilon_{0}\right)$. With the additional restriction to relativistic electrons, the observed Compton 

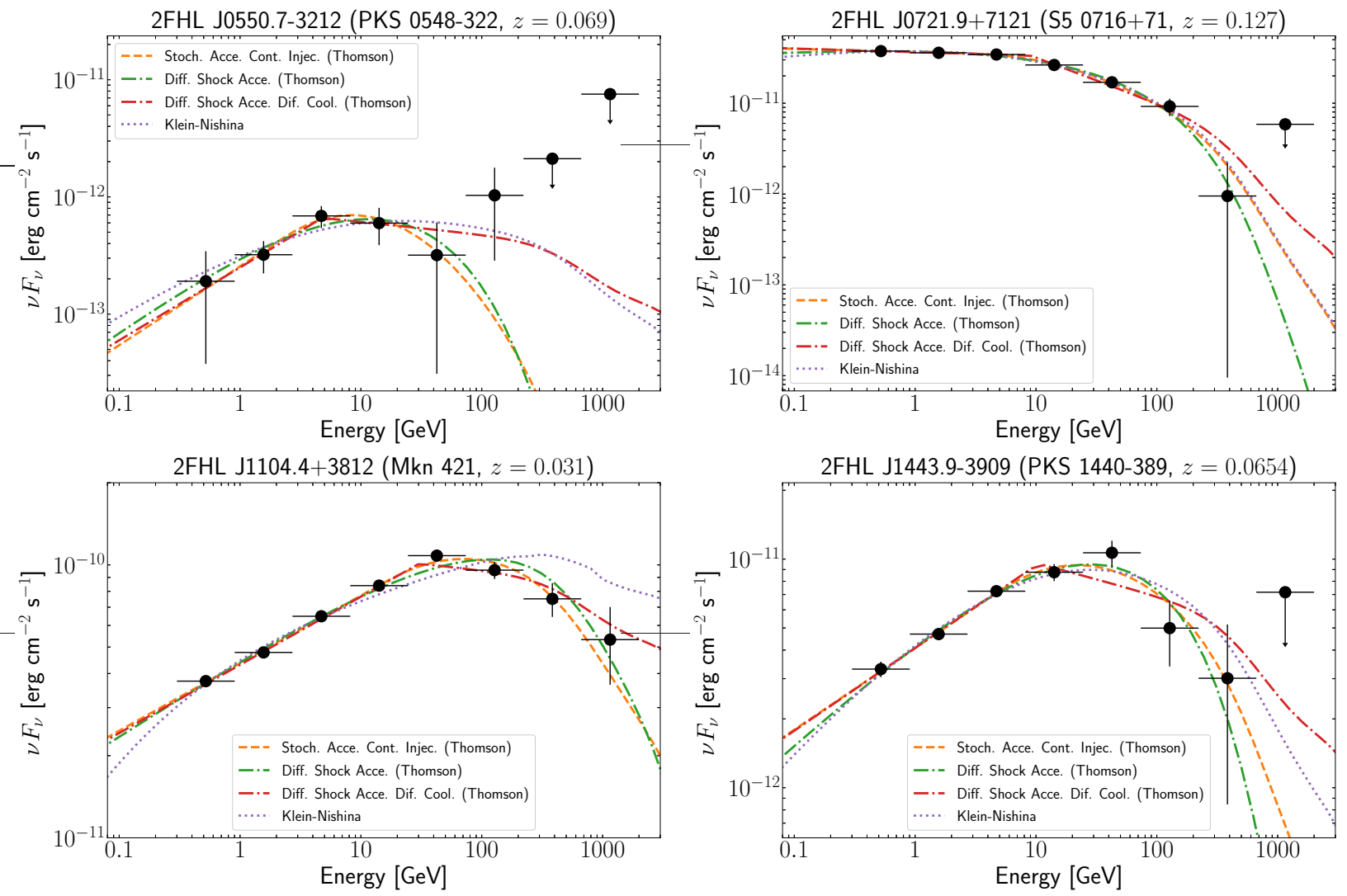

Figure 1. Examples of high-energy SEDs of four sources in our sample. The LAT data (black circles) are fitted to four emission models: stochastic acceleration with continuous injection in the Thomson regime (log-parabola with low-energy power law, dashed-orange line), radiation-reaction-limited first-order Fermi acceleration in the Thomson regime (power law with exponential cut-off, dashed-dotted green line), radiation-reaction-limited first-order Fermi acceleration with different cooling processes in the Thomson regime (broken power law, dashed-dotted red line), and first-order Fermi acceleration with Compton scattering in the Klein-Nishina regime (power law, dotted magenta line). The EBL attenuation is considered using the model presented by Domínguez et al. (2011). Notice that the apparent up-turn in the models at high energies are caused by transforming the models fitted to the intrinsic flux to the observed flux. This is due to the optical depth becoming almost constant at those energies for the given redshifts. (Note that the step like feature of the Klein-Nishina model for Mkn 421 is due to the numerical evaluation of the integral.)

flux is of the form

$$
\nu F_{\nu}\left(\epsilon_{\mathrm{sc}}\right)=A \epsilon_{\mathrm{sc}}^{2} \frac{n_{e}\left(\gamma_{0}\right)}{\sqrt{\epsilon_{\mathrm{sc}} \epsilon_{0}}}
$$

where $\gamma_{0}=\sqrt{\epsilon_{\mathrm{sc}} / \epsilon_{0}}$ and $A$ is a normalization constant. This implies that the observed flux will have a form similar to the electron distribution function.

\subsection{First-Order Fermi Acceleration with Thomson Scattering}

In the case of first-order Fermi acceleration (e.g., DSA; Kirk \& Heavens 1989; Ellison et al. 1990; Summerlin \& Baring 2012) a limiting process, such as radiative cooling and/or a decreasing chance for high-energy particles to cross the shock front a large number of times, gives rise to an electron distribution described by a power law with an exponential cut-off

$$
n_{e}\left(\gamma_{e}\right)=n_{e ; 0} \gamma_{e}^{-p} \exp \left(-\frac{\gamma_{e}}{\gamma_{c}}\right)
$$

where $p$ is the spectral index and $\gamma_{c}$ is the cut-off Lorentz factor. Substituting Eq. 2 into Eq. 1 and absorbing all constants into a proportionality constant $C_{1}$, the observed flux will have the form

$$
\nu F_{\nu}=C_{1} \nu^{-\alpha+1} \nu_{0}^{\alpha} \exp \left(-\sqrt{\frac{\nu}{\nu_{c}}}\right)
$$

where $\alpha=(p-1) / 2$ and $\nu_{c}=\gamma_{c}^{2} \nu_{0}$ is the cut-off frequency. This will be referred to as the power law with exponential cut-off (PL+EC) model. In practise, $C_{2}$ and $\nu_{0}$ cannot be constrained independently from a fit with this model, as they can be absorbed into a combined normalization constant $C_{2}^{\prime}=C_{2} \nu_{0}^{\alpha}$, and a given fit value of $\nu_{c}$ (within the Fermi range) can always be achieved for an appropriate combination of $\nu_{0}$ and $\gamma_{c}$, allowing for Compton scattering in the Thomson regime.

\subsection{Stochastic Acceleration with Continuous Injection and Thomson Scattering}


Tramacere et al. (2011) showed, using both a statistical and a diffusion equation approach, that stochastic acceleration gives rise to an electron distribution with a log-parabolic form, and by solving a diffusion equation with radiative losses, that the electron distribution resulting from stochastic acceleration with continuous injection could develop a low-energy power law tail while retaining a high-energy log-parabolic peak. Such an electron distribution could also result from a stochastic acceleration rate which is constant at lowenergies, but becomes energy dependent at higher energies (Massaro et al. 2006). Analytically, we have

$$
n_{e}\left(\gamma_{e}\right)=n_{e ; 0}\left\{\begin{array}{ll}
\left(\gamma_{e} / \gamma_{b}\right)^{-a} & \text { if } \gamma_{e} \leq \gamma_{b} \\
\left(\gamma_{e} / \gamma_{b}\right)^{-\left[a+b \ln \left(\gamma_{e} / \gamma_{b}\right)\right]} & \text { if } \gamma_{e}>\gamma_{b}
\end{array},\right.
$$

where $a$ is the low-energy limit of the slope, $b$ parameterizes the curvature of the distribution, and $\gamma_{b}$ is the Lorentz factor at which the break/transition occurs (see also Massaro et al. 2004; Tramacere et al. 2007). The curvature of the distribution is inversely proportional to both the number of acceleration steps or the acceleration time, and the variance in the energy gained during each acceleration step or the momentum diffusion coefficient. In the absence of radiative cooling, the distribution will therefore become a power law for very long acceleration times or effective momentum diffusion, but if radiative cooling is taken into account, the curvature will increase. Substituting Eq. 4 into Eq. 1, yields

$$
\nu F_{\nu}=C_{2} \nu \sqrt{\frac{\nu}{\nu_{0}}} \begin{cases}\left(\nu / \nu_{b}\right)^{-a / 2} & \text { if } \nu \leq \nu_{b} \\ \left(\nu / \nu_{b}\right)^{-\left[a+b \ln \left(\nu / \nu_{b}\right) / 2\right] / 2} & \text { if } \nu>\nu_{b}\end{cases}
$$

for the observed flux, where all constants were absorbed into the proportionality constant $C_{2}$ and $\nu_{b}=\gamma_{b}^{2} \nu_{0}$ is the frequency at which the break/transition occurs. Notice that there is a similar dependence between $\nu_{b}$ and $\nu_{0}$ as there is between $\nu_{c}$ and $\nu_{0}$ in the PL+EC model. This model will be referred to as the log-parabola with lowenergy power law $(\mathrm{LP}+\mathrm{PL})$ model.

\subsection{First-Order Fermi Acceleration with Different Acceleration / Cooling Regimes and Thomson Scattering}

If two different physical processes dominate in different energy ranges, such as radiative vs. adiabatic cooling, then the electron distribution can be described by a broken power-law:

$$
n_{e}\left(\gamma_{e}\right)=n_{e ; 0}\left\{\begin{array}{ll}
\left(\gamma_{e} / \gamma_{b}\right)^{-q} & \text { if } \gamma_{e} \leq \gamma_{b} \\
\left(\gamma_{e} / \gamma_{b}\right)^{-s} & \text { if } \gamma_{e}>\gamma_{b}
\end{array},\right.
$$

where $q$ and $s$ is the spectral index of the low- and highenergy power law, respectively. Substituting this into
Eq. 1 and absorbing all constants into a single proportionality constant, results in an observed flux with the form

$$
\nu F_{\nu}=C_{3} \nu \sqrt{\frac{\nu}{\nu_{0}}}\left\{\begin{array}{l}
\left(\nu / \nu_{b}\right)^{-q / 2} \text { if } \nu_{e} \leq \nu_{b} \\
\left(\nu / \nu_{b}\right)^{-s / 2} \text { if } \nu_{e}>\nu_{b}
\end{array} .\right.
$$

In this model, which will be referred to as the broken power-law (BPL) model, there is the same dependence between $\nu_{b}$ and $\nu_{0}$ as in the case of the LP+PL model.

\subsection{First-Order Fermi Acceleration with Klein-Nishina Scattering}

In the Klein-Nishina regime, the Compton cross section is more complicated, but for scattering by ultrarelativistic electrons, it can be well represented by the head-on approximation, in which the scattered photon propagates in the direction of the in-coming electron (see Böttcher et al. 2012; Dermer \& Menon 2012). Using the same setup as in the Thomson regime, the angle integrations can be done analytically to give the observed flux as a function of the normalized up-scattered photon energy, as done by Jones (1968) (see also Böttcher et al. 2012; Dermer \& Menon 2012). The decrease of the Compton cross section in the Klein-Nishina regime will lead to high-energy spectral curvature in the Compton spectrum even for an electron distribution described by a simple power law,

$$
n_{e}\left(\gamma_{e}\right)=n_{e ; 0} \gamma_{e}^{-p} .
$$

Again assuming a mono-energetic target-photon distribution and absorbing constant factors into a proportionality constant $C_{4}$, the observed Compton flux can be written as

$$
\begin{gathered}
\nu F_{\nu}=C_{4} \frac{\epsilon^{2}}{\epsilon_{0}} \int_{\gamma_{1}}^{\infty} \gamma_{e}^{-(p+2)} \times \\
{\left[2 q \ln (q)+(1+2 q)(1-q)+\frac{(1-q)\left(4 \epsilon_{0} \gamma_{e} q\right)^{2}}{2\left(1+4 \epsilon_{0} \gamma_{e} q\right)}\right] d \gamma_{e}}
\end{gathered}
$$

where

$$
q=\frac{\epsilon}{4 \epsilon_{0} \gamma_{e}\left(\gamma_{e}-\epsilon\right)},
$$

the subscript were dropped and it should be noted that only the up-scattering part of the integral of Jones (1968) relevant to the $\gamma$-ray regime is used. The limits on $\epsilon$ where the integral is non-zero, impose a lower limit on the $\gamma_{e}$ integration, given by

$$
\gamma_{1}=\frac{\epsilon+\sqrt{\epsilon^{2}+\epsilon / \epsilon_{0}}}{2} .
$$

This will be referred to as the Klein-Nishina (KN) model. 


\subsection{Influence of Relativistic Doppler Boosting}

In the case of external Compton (EC) scattering, relativistic Doppler boosting of the external photon fields into the rest frame of the emission region and back into the observer's frame needs to be considered. If the emission region moves with a bulk Lorentz factor $\Gamma=\left(1-\beta_{\Gamma}^{2}\right)^{-1 / 2}$ (typically with a value of $\Gamma \sim 10$ ) at an angle $\mu=\cos \theta_{\text {obs }}$ with respect to our line of sight, Doppler boosting is characterized by the Doppler factor $\delta_{D}=\left[\Gamma\left(1-\beta_{\Gamma} \mu\right)\right]^{-1}$. Blazars are observed at a small angle $\theta_{\text {obs }} \sim 1 / \Gamma$ with respect to the jet axis, so that the Doppler factor is typically of the order of the Lorentz factor, $\delta_{D} \approx \Gamma$. External target photons of energy $\epsilon_{0}$ are then Doppler boosted into the emission region rest frame (denoted here with primed quantities) as $\epsilon_{0}^{\prime} \approx \Gamma \epsilon_{0}$ and back into the observer's frame as $\epsilon_{\mathrm{sc}}^{\mathrm{obs}} \approx \Gamma \epsilon_{\mathrm{sc}}^{\prime}$. In the Thomson regime, we therefore have $\epsilon_{\mathrm{sc}}^{\mathrm{obs}} \approx \epsilon_{0} \Gamma^{2}\left(\gamma_{e}^{\prime}\right)^{2}$. Interpreting the values of $\epsilon_{0}$ resulting from the formalism developed above (neglecting Doppler boosting) and the fitting routine described below, as the actual value of the target photon energy (in the AGN rest frame), the values of $\gamma_{e}$ found in the fitting routine then correspond to co-moving electron energies of $\gamma_{e}^{\prime}=\gamma_{e} / \Gamma$.

In the case of $\mathrm{SSC}$, both the synchrotron target photons and the SSC-scattered high-energy photons are subject to the same Doppler boost $\delta_{D} \sim \Gamma$. Hence, the fit value of $\nu_{0}$ and $\epsilon_{0}$ may be interpreted as the observed synchrotron photon frequency and energy, respectively, and the Thomson limit applies when $\epsilon_{0} \gamma_{e} / \Gamma \ll 1$.

\subsection{Extragalactic Background Light Attenuation}

High-energy photons travelling over cosmological distances are attenuated by pair-production interactions with the extragalactic background light (EBL, Nikishov 1962; Gould \& Schréder 1966). The EBL is the diffuse infrared through ultraviolet radiation accumulated over the history of the Universe (e.g., Hauser \& Dwek 2001). The intrinsic flux is related to the observed flux by

$$
\left.\frac{d F}{d E}\right|_{\mathrm{int}}=\left.\frac{d F}{d E}\right|_{\mathrm{obs}} \exp [\tau(E, z)] .
$$

We consider this effect using the optical depths $\tau$ by Domínguez et al. (2011), which are provided as a function of observed $\gamma$-ray energy $E$ and redshift $z$ of the source.
The four models are fitted to the data using a $\chi^{2}$ minimization fitting routine. Upper limits $(1 \sigma)$ are also considered in the fitting by using half of the limit as both the flux data point and the flux error. This is a possible way to handle upper limits and thus, use as much spectral information as possible. Considering the large errors on flux points implied by the upper limits, the fitting routine will assign small weights to these data points. Therefore, we do not expect that the results would change qualitatively if the upper limits are treated differently.

In order to choose the best-fitting model we apply a maximum likelihood ratio test with a $95 \%$ confidence level. We use the logarithm of the likelihood ratio $t=-0.5\left(\chi_{1}^{2}-\chi_{0}^{2}\right)$ as the test statistic, where $\chi_{0}^{2}$ and $\chi_{1}^{2}$ are the chi-square values of the null and alternative models, respectively. The null model is the model with the larger number of degrees of freedom (DoF). Notice that the $\mathrm{PL}+\mathrm{EC}, \mathrm{LP}+\mathrm{PL}, \mathrm{BPL}$, and $\mathrm{KN}$ models have 4, 5, 5, and 3 free parameters, respectively, which means that with 8 flux data points, the models have 4, 3, 3, and 5 DoF, respectively. If the likelihood ratio is too large, the null model is rejected and the alternative model accepted, otherwise the null model is accepted and the alternative model rejected. Since $\Delta \chi^{2}$ is approximately $\chi^{2}$-distributed, with a DoF equal to the difference in the DoF of the two models being compared, a 95\% confidence level is equivalent to $t>0.5 \times 3.84=1.92$ for 1 DoF and $t>0.5 \times 5.99=2.995$ for 2 DoF. Notice that the $\mathrm{LP}+\mathrm{PL}$ and $\mathrm{BPL}$ models have the same number of DoF so that the likelihood ratio test cannot be performed on these two models. These two models are compared according to their $\chi^{2}$-values and the model with the smallest $\chi^{2}$-value is accepted as the favoured model. This test is done for each blazar between all the different combinations of models. The model that was preferred when compared to all other models is then accepted as the favoured model. Obviously, for any individual blazar, this cannot be considered a statistically robust statement of preference for a certain model. However, a systematic preference of one model throughout the sample of 128 blazars that we have investigated here, would provide a clear indication concerning the true spectral shape.

\section{FITTING METHODOLOGY}


Table 1. Rejection criteria applied to fits and the number of fits rejected. See text for motivation of these rejection criteria.

\begin{tabular}{|c|c|c|c|c|c|c|c|}
\hline Model & Rejection Criteria & All (128) & Variable (47) & Non-variable (81) & BL Lacs (106) & FSRQs (10) & Other (12) \\
\hline \multirow{3}{*}{$\mathrm{PL}+\mathrm{EC}$} & $Q<0.001$ & 2 & 2 & 0 & 2 & 0 & 0 \\
\hline & $\alpha<0.5$ & 19 & 1 & 18 & 18 & 0 & 1 \\
\hline & Total rejected & 21 & 3 & 18 & 20 & 0 & 1 \\
\hline \multirow{4}{*}{$\mathrm{LP}+\mathrm{PL}$} & $Q<0.001$ & 1 & 1 & 0 & 0 & 1 & 0 \\
\hline & $a<1$ & 1 & 0 & 1 & 1 & 0 & 0 \\
\hline & $b<0$ & 34 & 6 & 28 & 26 & 1 & 7 \\
\hline & Total rejected & 36 & 7 & 29 & 27 & 2 & 7 \\
\hline \multirow{4}{*}{$\mathrm{BPL}$} & $Q<0.001$ & 3 & 2 & 1 & 1 & 1 & 1 \\
\hline & $q<2$ & 21 & 2 & 19 & 19 & 0 & 2 \\
\hline & $s<q$ & 30 & 6 & 24 & 25 & 1 & 4 \\
\hline & Total rejected & 54 & 10 & 44 & 45 & 2 & 7 \\
\hline \multirow{4}{*}{$\mathrm{KN}$} & $Q<0.001$ & 6 & 6 & 0 & 3 & 2 & 1 \\
\hline & $p<1$ & 12 & 2 & 10 & 10 & 0 & 2 \\
\hline & $\epsilon_{0}>1 \times 10^{-4}$ & 69 & 30 & 39 & 50 & 10 & 9 \\
\hline & Total rejected & 71 & 32 & 39 & 52 & 10 & 9 \\
\hline
\end{tabular}

\section{RESULTS}

In this Section, the results of the fits of the four physically motivated models to the 2FHL blazar data are presented.

\subsection{Accepted models}

In some cases, even though formally an acceptable spectral fit could be achieved with a given model, the best-fit parameters are problematic and/or unphysical. We expect $\gamma$-ray emission produced by radiatively cooled electrons. Thus, a radiation spectrum indicating an electron spectrum harder than $\gamma^{-2}$ would have to be accelerated/injected from a population following a spectrum harder than $\gamma^{-1}$, which is difficult to reconcile with any known particle acceleration mechanism. It is highly unlikely that the spectra of the PL+EC, LP+PL, BPL, and $\mathrm{KN}$ models have a photon spectral index $\alpha$ harder than 0.5 , or a spectral index of the radiating particle distribution of $a<1, q<2$, or $p<1$, respectively. We point out that we have assumed that the electron spectra do not have low-energy cut-offs, i.e., our electron spectra always start at $\gamma_{\text {min }}=1$. While a large value of $\gamma_{\min } \gg 1$ could, in principle, also produce very hard low-energy $\gamma$-ray spectra (see, e.g., Katarzyń ski 2012), there is no accepted scenario which would realistically produce such a large low-energy cut-off. We have therefore not considered this possibility in this study.

There are a few cases for the LP+PL model where $b$ have negative values, causing the fitted spectra to curve upwards, which is not physical. There is a large number of fitted values for $\epsilon_{0}$ that fall in the Thomson regime. In the other models, $\nu_{0}$ enters only as an arbitrary normalization constant due to the dependence of $\gamma_{c}$ or $\gamma_{b}$ on $\nu_{0}$, which could be absorbed into $C$; this was not done in order to eliminate a dependence of $C_{1}$ on $\alpha$ in the case of the PL $+\mathrm{EC}$ model or a dependence between $C$ and $\nu_{b}$ in the case of the LP+PL and BPL models. The fitted value might therefore be considered unphysical if $\epsilon_{0}>1 \times 10^{-4}$ for the KN model. For the BPL model we additionally require that the high-energy component is softer than the low-energy component and hence $s<q$ must hold. The probability for the $\chi^{2}$-value to be larger than a certain $\chi^{2}$-value by chance, $Q$, was also calculated for each fit. If $Q \gtrsim 0.1$, the fit is believable; if $0.1 \gtrsim Q \gtrsim 0.001$, the fit may be acceptable if the uncertainties are not normally distributed or have been moderately underestimated; if $Q \lesssim 0.001$, then the fit can be statistically rejected; if $Q$ is very close to 1 , the fit might be too good to be true and this can be caused by an overestimation of the uncertainties or fraud in the data points. However, since the proportionality constants $C_{i}$ are arbitrary, the latter case can only be interpreted as a good fit.

Based on these criteria some of the fits were rejected, as summarized in Table 1. The average with standard deviation for the fitted parameters of the four models to the SEDs are summarized in column three to eight of Table 2 and the summary of the fitted parameters of 
Table 2. Averages of the model parameters of the fits, as well as the range of $\chi_{r}^{2}$-values for comparison. See the text for details.

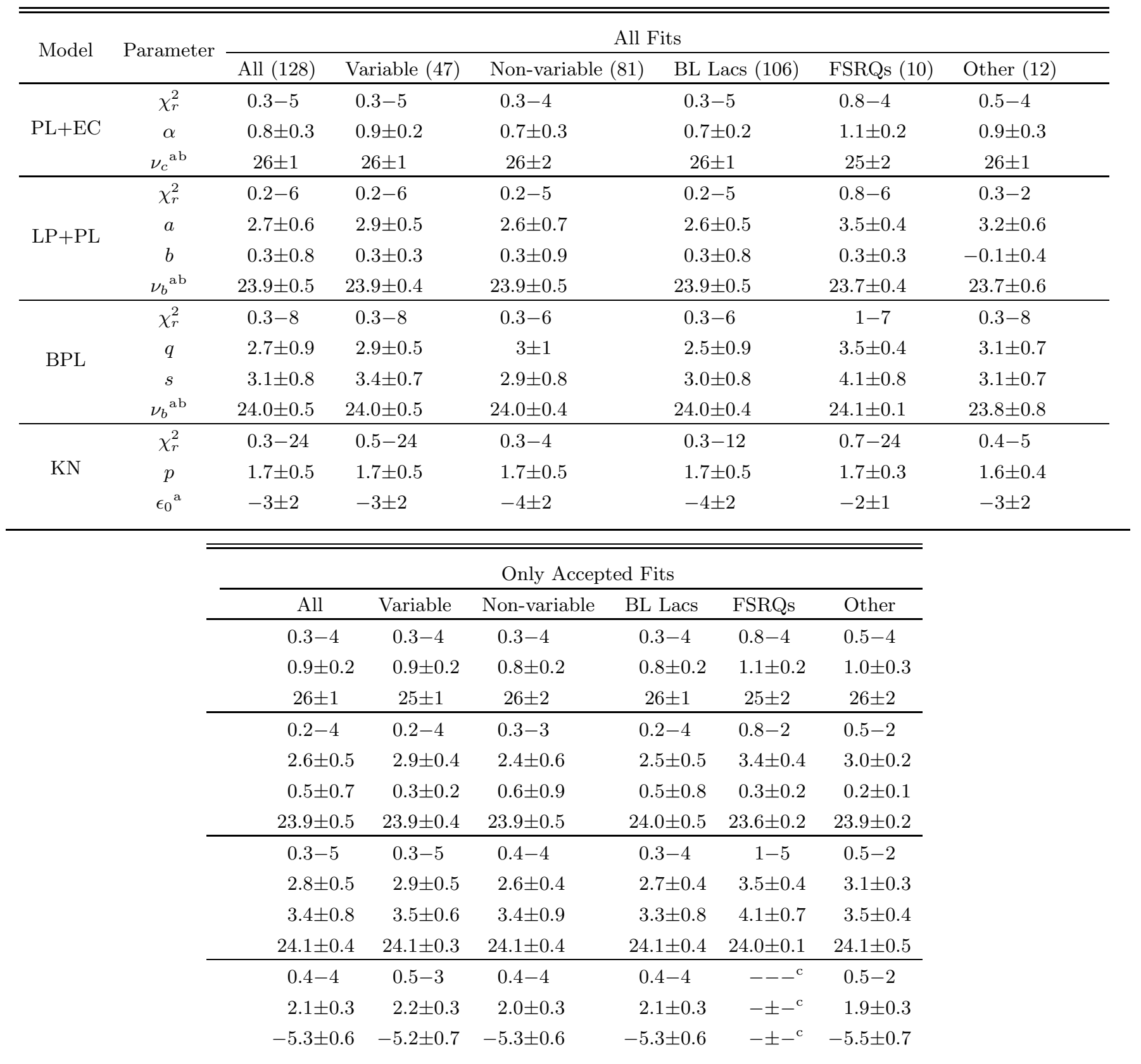

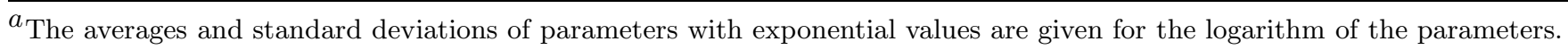
$b_{\mathrm{Hz}}$

${ }^{c}$ Only one or no accepted model.

only the accepted fits are given in the last six columns of Table 2. The range of reduced chi-squared values $\chi_{r}^{2}$ are also included in the table for comparison while the normalization constants and $\nu_{0}$ of the PL+EC, LP+PL, and BPL models are not shown since they are arbitrary and not of interest. The average and standard deviation of parameters with exponential values $\left(\nu_{c}, \nu_{b}\right.$ and $\left.\epsilon_{0}\right)$ are given as the average and standard deviation of the base
10 logarithm of the parameters.

The restrictions that the high-energy component of the BPL model is softer than the low-energy component and that Compton scattering occur indeed in the Klein-Nishina regime in the KN model, have led to the rejection of a lot of the fits of these models. The frequencies corresponding to $\epsilon_{0}$ for the accepted KN fits are of the order of $\sim 10^{16}-10^{17} \mathrm{~Hz}$ and fall in the ultraviolet 
to soft X-ray range, characteristic of synchrotron photons in the case of ISP or HSP blazars. The validity of the PL+EC model with Compton scattering in the Thomson regime up to $\sim 1 \mathrm{TeV}$ implies that the target photons must have frequencies $\nu_{0} \lesssim 10^{14} \mathrm{~Hz}$, favouring the dust-torus emission as their source. In several fits of the PL+EC model, very large values of the cut-off frequency $\nu_{c}$ (up to $\sim 10^{31} \mathrm{~Hz}$, compared to the data ranging up to $\sim 3 \times 10^{27} \mathrm{~Hz}$ ) resulted, indicating that the fit could be well approximated by a pure power law. This is also seen in the LP+PL model as small curvature parameter $b$ values.

\subsection{Variable and Non-variable Blazars}

The physical processes underlying the four models are quite different and most likely time-dependent. It might be expected that stochastic acceleration would always be present if there is turbulence in the jet and the decrease of the Compton cross section in the KleinNishina regime will be relevant whenever the target photon energy is $\epsilon_{0} \gtrsim 10^{-5}$ in which case $\gamma$-ray photons of $>10 \mathrm{GeV}$ can no longer be produced by Thomson scattering. However, the relevant acceleration and cooling processes are highly time-dependent and a combination of all of these processes could lead to artificial spectral features in the time-averaged spectra which we are fitting. In an attempt to avoid such complications, the blazars were divided into variable and nonvariable blazars. Unfortunately, $2 \mathrm{FHL}$ presents a variability analysis only considering photons above $50 \mathrm{GeV}$, not for our broader energy range data $(300 \mathrm{MeV}-2 \mathrm{TeV})$. Developing a complete time analysis for the data is beyond the scope of this paper. However, we can work around this limitation by using the $3 \mathrm{FHL}$ variability study (Ajello et al. 2017) since the 3FHL contains 127 of the 128 2FHL sources (the only drawback is that photons below $10 \mathrm{GeV}$ are not considered in the variability analysis). According to the 3FHL catalog there are 47 variable blazars in our sample and we assume that the other 81 blazars are non-variable or nearly so.

The average with standard deviation for fitted parameters of the four models of the variable and non-variable blazars are summarized in columns 4 and 5 of Table 2, respectively. Also shown in columns 6,7 , and 8 , is a summary of the fitted parameters for BL Lacs, FSRQs, and other blazar types, respectively. The numbers of fits rejected by the various rejection criteria are also summarized in Table 1. Lower spectral indices (harder spectra) are needed to fit non-variable blazars than variable ones and BL Lacs also require lower spectral indices than other blazar classes. Essentially, most of the fits rejected due to unphysically hard spectra are those of non-variable and BL Lac blazars. These trends also appear when comparing the averages of the fitted param- eters and qualitatively, the average values of the fitted parameters do not differ much between the subgroups of the accepted fits. The KN model seems incapable of reproducing the spectra of FSRQs and blazars of other types.

\subsection{Preferred Model}

Qualitatively, when comparing the four models in Fig. 1 and the $\chi_{r}^{2}$-values in Table 2, it seems that all four models fit the SEDs similarly well. The number of times each model provided the best fit, based on the likelihood ratio test outlined in Section 4 and where a model was counted as being a good fit if the other three models were rejected, should quantitatively indicate which model may be considered systematically preferred. These results are summarized in Table 3.

Focussing only on the accepted fits, the LP+PL and BPL models seem to be systematically disfavoured for most blazars. This indicates strong evidence against Thomson scattering by a log-parabola or broken power law electron distribution. The PL+EC model was preferred for the majority of the variable blazars, the FSRQs in the sample, as well as for blazars of unknown type (other). For the non-variable blazars as well as for BL Lac type blazars, the PL+EC and KN models were preferred approximately equally often, with a slight preference for the PL+EC model.

\section{SUMMARY AND DISCUSSION}

In this work we analyzed the first nine years of FermiLAT data in the energy range from $300 \mathrm{MeV}$ to $2 \mathrm{TeV}$ in order to extend the energy spectral coverage of the 128 2FHL blazars. These spectral data were compared to four models for the production of $\gamma$-ray spectra assuming a single-zone leptonic model: (1) radiation-reactionlimited first-order Fermi acceleration of electrons (power law with exponential cut-off) with Compton scattering in the Thomson regime, (2) stochastic acceleration of electrons with continuous injection (log-parabola with low-energy power law) and Compton scattering in the Thomson regime, (3) first-order Fermi acceleration of electrons with different acceleration/cooling mechanisms dominating in different energy regimes (broken power law) and Compton scattering in the Thomson regime, and (5) Compton scattering by a pure power law distribution of electrons with spectral curvature due to scattering in the Klein-Nishina regime.

Obviously, these are not the only plausible spectral shapes. However, they represent four fundamentally different, physically plausible ways of the formation of $\gamma$-ray spectra in blazars, and there is no (finite) exhaustive list of all possible combinations of effects that might contribute in reality. The $\mathrm{PL}+\mathrm{EC}, \mathrm{LP}+\mathrm{PL}$, and BPL models, corresponds to physically motivated elec- 
Table 3. Number of times each model fitted the best.

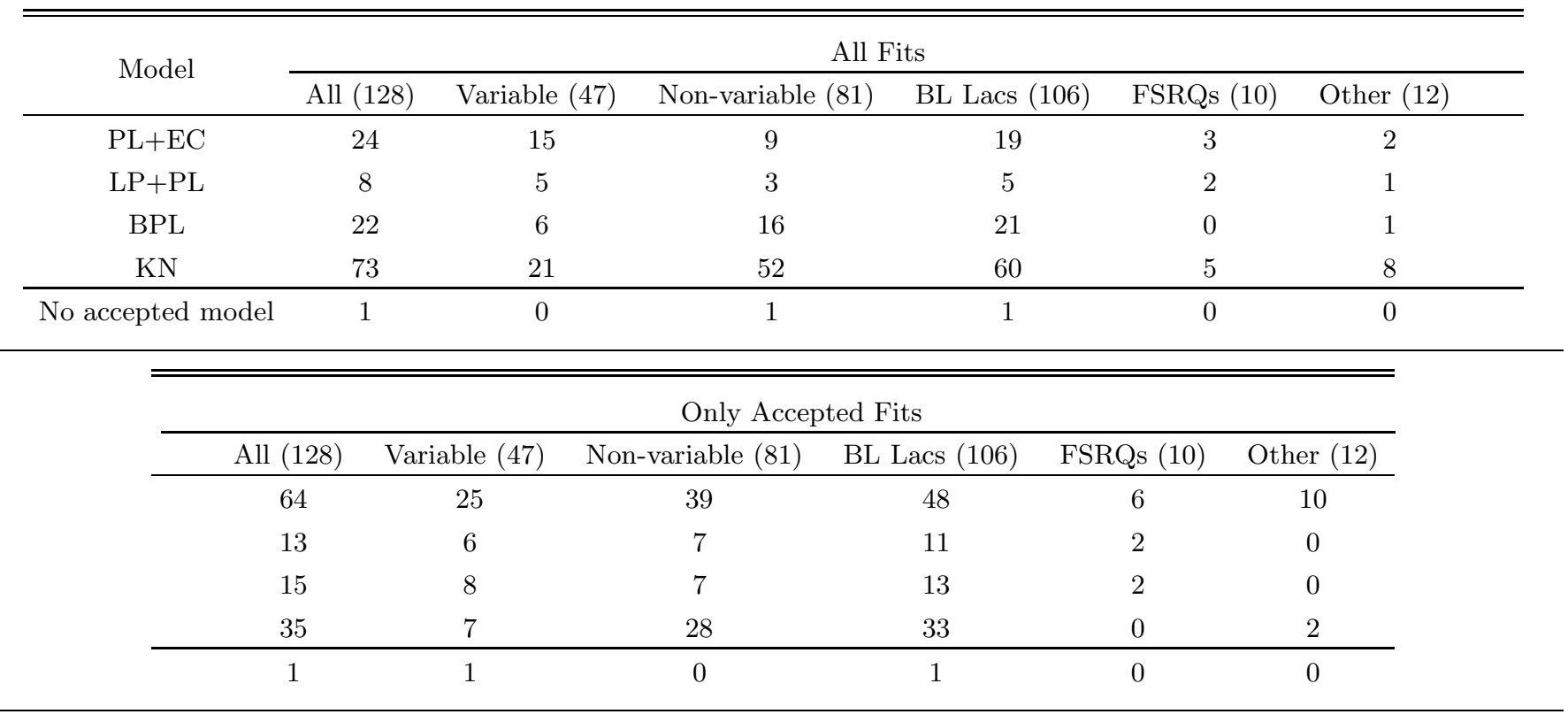

tron distributions (DSA, stochastic acceleration with continuous injection, and energy dependent acceleration/cooling, respectively), assuming Compton scattering in the Thomson regime, and the pure power law would simply be extreme cases of either model $(b=0$, $\nu_{c} \rightarrow \infty$, or $\nu_{b} \rightarrow \infty$, respectively). The power law with Compton scattering in the Klein-Nishina regime was introduced to check whether Klein-Nishina effects might, instead, be dominant in the formation of spectral curvature. We therefore consider these four shapes "basic building blocks" of the spectral shapes of blazars. A (more realistic) combination of LP or PL+EC with Klein-Nishina effects would introduce too many free parameters, so that the available spectra would not be able to provide a meaningful distinction. A systematic preference for any (one or two) of these fundamental models throughout the entire 2FHL sample may be considered a significant indication of the dominant mode of $\gamma$-ray spectra formation in these blazars.

The fitted parameters found here only refer to the general shape of the high-energy spectrum and constrain the energy of target photons for Compton scattering and the energy distribution of the electrons. However, other physical parameters (such as, e.g., the magnetic field or the bulk Lorentz and Doppler factor), cannot be meaningfully constrained based on fits to the $\gamma$-ray spectra alone. This degeneracy can be broken by fitting a broader energy range, including the synchrotron component of the SED (see, e.g., Paliya et al. 2018, for an application). While the shape of the high-energy tail of the synchrotron spectrum can often be probed well in HSP blazars (where the synchrotron peak is often prominent in the X-ray regime), this is generally difficult in
LSP and ISP blazars (Abdo et al. 2010b) (where the synchrotron peak is located in the infrared though optical regime and the high-energy tail is often unobservable), as it can be located in the inaccessible ultraviolet regime and/or because it is overwhelmed by the lowenergy tail of the high-energy spectral component. It is therefore difficult to characterize the full SED. Thus, the large sample of well-determined blazar $\gamma$-ray spectra measured by Fermi-LAT seems to provide the best and most abundant test bed for the high-energy shapes of blazar spectra, even though it only allows us to characterize the underlying physical processes and not to pin down specific parameter values.

The blazars were divided into a variable and nonvariable subgroup, as a combination of the different, time-dependent physical processes could lead to artificial spectral features in the time-averaged spectra of variable blazars. The blazars were also further divided into BL Lacs, FSRQs, and other types of blazars, as the physical acceleration mechanisms could vary among the different types of blazars. Our most significant result is the rejection of the model with Thomson scattering by an electron distribution with a broken power law or a log-parabola with a low-energy power law. This does not imply a complete rejection of these electron distributions. However, it indicates that, if such an electron distribution is present, additional effects, such as the Klein-Nishina cut-off, must play a significant role in the formation of blazar $\gamma$-ray spectra.

The first-order Fermi acceleration with Thomson scattering and the decrease of the Compton cross section in the Klein-Nishina regime could successfully explain the high-energy spectral shape of almost equal numbers of 
non-variable blazars as well as of BL Lacs. This is consistent with the standard interpretation of SSC-dominated $\gamma$-ray emission in BL Lac objects, where a gradual transition from the Thomson to the Klein-Nishina regime is expected throughout the high-energy $\gamma$-ray range. We remind the reader that for the $\mathrm{PL}+\mathrm{EC}$ model, the target photon energy cannot be constrained from the spectral fits to $\gamma$-ray spectra alone, as there is a degeneracy between $\nu_{0}$ and $\gamma_{c}$ (see Section 3.2). Combinations of $\nu_{0}$ and $\gamma_{c}$ can therefore always be found that allow for Compton scattering in the Thomson regime up to the highest Fermi energies. This requires electron cut-off energies of $\gamma_{c} \gtrsim 10^{5}$ and soft target photons with frequencies $\nu_{0} \lesssim 10^{14} \mathrm{~Hz}$, thus strongly favouring a dusttorus origin of the target photons (in agreement with the results by Costamante et al. 2018).

Although DSA might be expected as a plausible acceleration mechanism for variable blazars, there might be a contribution of various physical mechanisms to the spectral shape of variable blazars, as mentioned previously. It is indeed plausible that the spectrum could be described by a combination of different processes and not just a single electron distribution as assumed in each model. In particular, spectral curvature may be a combination of both a curved electron distribution and Klein-Nishina effects at the same time. It is also possible for the Klein-Nishina effects to affect the electron distribution (e.g., Moderski et al. 2005). If the electron distribution is a power law which is hardened by inefficient Compton cooling at the high-energy end (if Compton cooling strongly dominates over other radiative cooling mechanisms), then this would result in a power law photon spectrum, which is inconsistent with most Fermi-LAT spectra investigated here.

The assumption of mono-energetic target photon spectra may also be an over-simplification, as broad nonthermal target photon distributions may result in additional spectral curvature (see, e.g., Tavecchio et al. 1998). It is well known that Compton scattering of a broad non-thermal synchrotron spectrum by a broad non-thermal electron distribution introduces additional curvature, which is primarily caused by Klein-Nishina effects at high energies (which we are interested in here). As our results are well consistent with the standard paradigm that SSC dominates for BL Lacs, introducing the additional complication of SSC with a broad target photon spectrum would likely not yield any additional insights. For thermal target photon fields, however, the Compton spectrum is only weakly dependent on the dis-

\footnotetext{
1 Any opinion, finding and conclusion or recommendation expressed in this material is that of the authors, and the NRF does
}

tribution of seed photons, but depends critically on their characteristic energy, which is fitted within physically reasonable limits.

The best-fit values of $\nu_{0} \sim 10^{16}-10^{17} \mathrm{~Hz}$ for the $\mathrm{KN}$ model is compatible with the synchrotron emission from ISP and HSP blazars, thus favouring the SSC hypothesis. In the case of FSRQs, which are best fitted by a $\mathrm{PL}+\mathrm{EC}$ in the Thomson regime, our results favour $\gamma$ ray emission scenarios based on Compton scattering of infrared radiation from the dust torus. This result is interesting for $\mathrm{TeV}$ telescopes since it will be possible for them to detect more FSRQs than if external photons were provided by the BLR. Indeed, very high energy measurements $(E>100 \mathrm{GeV})$ with these telescopes, especially with the future Cherenkov Telescope Array, will help in characterizing the blazar $\gamma$-ray emission.

The Fermi-LAT Collaboration acknowledges generous ongoing support from a number of agencies and institutes that have supported both the development and the operation of the LAT as well as scientific data analysis. These include the National Aeronautics and Space Administration and the Department of Energy in the United States, the Commissariat à l'Energie Atomique and the Centre National de la Recherche Scientifique / Institut National de Physique Nucléaire et de Physique des Particules in France, the Agenzia Spaziale Italiana and the Istituto Nazionale di Fisica Nucleare in Italy, the Ministry of Education, Culture, Sports, Science and Technology (MEXT), High Energy Accelerator Research Organization (KEK) and Japan Aerospace Exploration Agency (JAXA) in Japan, and the K. A. Wallenberg Foundation, the Swedish Research Council and the Swedish National Space Board in Sweden.

Additional support for science analysis during the operations phase is gratefully acknowledged from the Istituto Nazionale di Astrofisica in Italy and the Centre National d'Études Spatiales in France. This work performed in part under DOE Contract DE-AC02$76 \mathrm{SF} 00515$.

The authors thank Markos Georganopoulos and the anonymous reviewer for useful comments. JPvdB acknowledges the support of the National Astrophysics and Space Science Program (NASSP) in South Africa. MB acknowledges support through the South African Research Chair Initiative (SARChI) of the Department of Science and Technology and the National Research Foundation $^{1}$ (NRF) of South Africa under NRF SARChI Chair grant no. 64789. AD thanks the support of the Juan de la Cierva program from the Spanish MEC.

not accept any liability in this regard. 


\section{REFERENCES}

Aartsen, M. G., et al., 2018a, Science, 361, 1378

Aartsen, M. G., et al., 2018b, Science, 361, 147

Abdo, A.A., Ackermann, M., Ajello, M., et al. 2010a, ApJ, 710, 1271

Abdo, A.A., Ackermann, M., Ajello, M., et al. 2010b, ApJ, 716, 30

Acero, F., Ackermann, M., Ajello, M., et al. 2015, ApJS, 218, $23 \mathrm{~A}$

Ackermann, M., Ajello, M., Atwood, W.B., et al. 2016, ApJS, 222, 5

Ajello, M., Atwood, W.B., Baldini, L., et al. 2017, ApJS, 232, 18

Böttcher, M., 2007, Astrophys. \& Space Science, 309, 95

Böttcher, M., Harris, D.E. \& Krawczynski, H. 2012,

Extragalactic Jets from Active Galactic Nuclei (Chichester: Springer-Praxis)

Böttcher, M., Reimer, A., Sweeney, K. \& Prakash, A. 2013, ApJ, 768,54

Costamante, L., Cutini, S., Tosti, G., et al. 2018, in prep.

Dermer, C.D. \& Menon, G. 2009, High Energy Radiation from Black Holes: Gamma Rays, Cosmic Rays, and Neutrinos (New Jersey: Princeton University)

Domínguez, A., Primack, J.R., Rosario, D.J., et al. 2011, MNRAS, 410, 2556

Domínguez, A. \& Ajello, M., 2015, ApJ, 813, L34

Ellison, D.C., Jones, F.C. \& Reynolds, S.P. 1990, ApJ, 360, 702

Fossati, G., Maraschi, L., Celotti, A., Comastri, A., Ghisellini, G. 1998, MNRAS, 299, 433
Ghisellini, G., Righi, C., Costamante, L. \& Tavecchio, F. 2017, MNRAS, 469, 255G

Gould, R.J. \& Schréder, G. 1966, PhRvL, 16, 252

Hauser, M.G. \& Dwek, E. 2001, ARA\&A, 39, 249

Jones, F.C. 1968, Phys. Rev., 167, 1159

Katarzyń ski, K., 2012, A\&A, 537, A47

Kirk, J.G. \& Heavens, A.F. 1989, MNRAS, 239, 995

Landau, R., Golisch, B., Jones, T.J., et al. 1986, ApJ, 308, 78

Massaro, E., Perri, M., Giommi, P., \& Nesci, R. 2004, A\&A, 413, 489

Massaro, E., Tramacere, A., Perri, M., Giommi, P., \& Tosti, G. 2006, A\&A, 448, 861

Moderski, R., Sikora, M., Coppi, P.S. \& Aharonian, F. 2005, MNRAS, 363, 954

Nikishov, A.I. 1962, Sov. Phys. JETP, 14, 393

Paliya, V.S., Zhang, H., Böttcher, M., Ajello, M., Domínguez, A., Joshi, M., Hartmann, D., \& Stalin, C.S., 2018, ApJ, 863, 98

Summerlin, E.J. \& Baring, M.G. 2012, ApJ, 745, 63

Tavecchio, F., Maraschi, L. \& Ghisellini, G. 1998, ApJ, 509, 608

Tramacere, A., Massaro, F., \& Cavaliere, A. 2007, A\&A, 466, 521

Tramacere, A., Massaro, E., \& Taylor, A.M. 2011, ApJ, 739, 66

Urry, C.M. \& Padovani, P. 1995, PASP, 107, 803 